\title{
Tomographic reconstruction of the ionospheric electron density as a function of space and time
}

\author{
Onur Erturk ${ }^{a}$, Orhan Arikan ${ }^{a}$, Feza Arikan ${ }^{b, *}$ \\ ${ }^{a}$ Bilkent University, Department of Electrical and Electronics Engineering, Bilkent Ankara 06800, Turkey \\ ${ }^{\mathrm{b}}$ Hacettepe University, Department of Electrical and Electronics Engineering, Beytepe Ankara 06800, Turkey
}

Received 23 November 2007; received in revised form 2 July 2008; accepted 18 August 2008

\begin{abstract}
Electron density distribution is the major determining parameter of the ionosphere. Computerized Ionospheric Tomography (CIT) is a method to reconstruct ionospheric electron density image by computing Total Electron Content (TEC) values from the recorded Global Positioning Satellite System (GPS) signals. Due to the multi-scale variability of the ionosphere and inherent biases and errors in the computation of TEC, CIT constitutes an underdetermined ill-posed inverse problem. In this study, a novel Singular Value Decomposition (SVD) based CIT reconstruction technique is proposed for the imaging of electron density in both space (latitude, longitude, altitude) and time. The underlying model is obtained from International Reference Ionosphere (IRI) and the necessary measurements are obtained from earth based and satellite based GPS recordings. Based on the IRI-2007 model, a basis is formed by SVD for the required location and the time of interest. Selecting the first few basis vectors corresponding to the most significant singular values, the 3-D CIT is formulated as a weighted least squares estimation problem of the basis coefficients. By providing significant regularization to the tomographic inversion problem with limited projections, the proposed technique provides robust and reliable 3-D reconstructions of ionospheric electron density.
\end{abstract}

(C) 2009 COSPAR. Published by Elsevier Ltd. All rights reserved.

Keywords: Ionosphere; Ionospheric reconstruction; Computerized Ionospheric Tomography (CIT); Ground Positioning Satellites (GPS); Occultation data; Singular Value Decomposition (SVD); Voxelization

\section{Introduction}

Estimation of electron density distribution of the ionosphere as a function of space and time is a challenging problem due to variability in space and time. Computerized Ionospheric Tomography (CIT) is a method to reconstruct ionospheric electron density image by computing Total Electron Content (TEC) values from the earth and satellite based recorded GPS signals. Due to the multi-scale variability of the ionosphere and inherent biases and errors in the computation of TEC, CIT constitutes an underdetermined ill-posed inverse problem. GPS satellites and receiv-

\footnotetext{
* Corresponding author.

E-mail addresses: oarikan@ee.bilkent.edu.tr (O. Arikan), arikan@ hacettepe.edu.tr (F. Arikan).
}

ers provide Total Electron Content (TEC) measurements along a network of lines connecting satellites to the receivers. Therefore, a line-projection relates the electron density distribution to the available measurements resulting in a tomographic set up for the estimation problem. However, the classical tomographic reconstruction techniques fail to provide reliable results with the limited number of available line-projections.

In addition, the time varying nature of the electron density distribution creates further complications. Ionospheric imaging of electron density distribution has four dimensions in latitude, longitude, altitude (height) and time. Computerized Ionospheric Tomography is of utmost interest in recent years. Various approaches for the solution of the CIT include serial expansion of electron density into two dimensional basis functions, iterative algebraic 
reconstruction methods, neural network and statistical analysis methods. Reconstruction of the ionosphere by computerized tomography is first studied by Austen et al. (1988) using the Algebraic Reconstruction Technique (ART) which provides a 2-D reconstruction of the ionosphere. One of the first application of ART is performed by Pryse and Kersley (1992). A major group of studies use Abel Inversion Technique in the reconstruction (Hajj and Romans, 1998; Tsai et al., 2001). The reconstructions using this technique are improved by Garcia Fernandez et al. (2003a) by using the IRI model. In a later study by Garcia Fernandez et al. (2003b), ionosonde and GPS data are used for CIT. Hansen et al. (1997) applied computerized tomography using Radon transformation. In recent years, space and time reconstructions of the ionosphere are studied. However, the classical tomographic reconstruction techniques fail to provide reliable estimates due to the limited number of available line integrals. Furthermore, the time varying nature of the electron density creates challenging complications. Novel CIT reconstruction techniques are proposed in Arikan et al. $(2007 \mathrm{a}, \mathrm{b})$ using Random Field Priors and basis functions from the set of Squeezed Legendre Polynomials, truncated Legendre Polynomials, Haar Wavelets and singular value decomposition (SVD) with IRI model. In Arikan et al. (2007b), it is shown that best results are obtained for the basis functions from the model itself through SVD.

In recent years, GPS dual frequency signals have been widely used to estimate both regional and global TEC values (Komjathy, 1997; Liao, 2000). The TEC computation methods and their advantages and disadvantages are widely discussed in the literature (Jakowski et al., 1996; Liao, 2000;Arikan et al., 2003). Regularized Estimation of TEC (Reg-Est) is a technique for estimation of high resolution, reliable and robust TEC estimation as discussed in detail by Arikan etal. (2003, 2004, 2007). In a study conducted by Arikan et al. (2003), regularized estimation of vertical total electron content from Global Positioning System data is researched and a new method is proposed. The GPS data used in this study is obtained by the developed Reg-Est method discussed in Nayir et al. (2007) and IONOLAB using the phase delay measurements.

In this study, to improve the reliability of the obtained 3-D estimates, we propose an SVD based tomographic reconstruction technique, where the IRI-2007 electron density profiles are used as a model and an a priori source of information based on the 2-D CIT reconstruction method and results discussed in Arikan et al. (2007b). To improve the performance of the reconstruction, we form a basis by using SVD of a matrix whose columns are generated from the IRI-2007 model for the required location and the time of interest. Also, to account for the variation as a function of solar activity, we consider IRI-2007 electron density profiles with similar sun-spot number index. The SVD basis varies significantly with respect to time of the day, and day of the year. Therefore, a reconstruction based on a fixed basis would have limited applicability around the
Earth with respect to time. Hence, the basis components should be updated in time. Although it will not be detailed in this paper, we observed that hourly updates on the reconstruction basis yield acceptable performance. We also investigated reconstruction quality of the proposed technique on synthetic measurements showing that robust estimation of the ionospheric electron density distribution that fits to the observed data as well as the IRI-2007 model is possible.

In Section 2, the proposed SVD basis approach for the ionospheric reconstruction is introduced. Section 3 contains the application of the proposed CIT algorithm on synthetic and model based electron density distributions.

\section{Proposed approach for ionospheric reconstruction}

The proposed SVD approach for the tomographic reconstruction of the ionospheric electron density in both space and time is discussed below. The first part of the section introduces the measurement model. The voxelization and basis formation are explained in detail in the second part. The third subsection discusses the reconstruction based on the proposed SVD approach.

\subsection{Measurement model}

STEC measurement model for GPS satellites can be closely approximated as a line integral between the satellite and receiver positions given by:

$y_{u, m}\left(t_{0}\right)=\int_{0}^{1} e\left(x_{u}+d_{x} \lambda, y_{u}+d_{y} \lambda, z_{u}+d_{z} \lambda, t_{0}\right) d_{u, m} d \lambda$,

where $d_{x}, d_{y}$ and $d_{z}$ denote the difference between the coordinates of GPS satellite $m$ and GPS receiver $u$; satellite $m$ has Cartesian coordinates $\left(x_{m}, y_{m}, z_{m}\right)$; receiver $u$ has Cartesian coordinates $\left(x_{u}, y_{u}, z_{u}\right)$ at time $t_{0}$ and

$d_{u, m}=\sqrt{\left(x_{u}-x_{m}\right)^{2}+\left(y_{u}-y_{m}\right)^{2}+\left(z_{u}-z_{m}\right)^{2}}$.

This expression can be applied to occultation data by replacing GPS receiver coordinates with that of the LEO satellite coordinates.

\subsection{Voxelization and basis formation}

The reconstruction is implemented on a grid structure over the ionosphere made up of prisms that extend in latitude, longitude and altitude. Each prism is called a voxel and in total there are $N_{r} \times N_{\theta} \times N_{\phi}$ voxels considered in reconstruction problem, where $N_{r}, N_{\theta}$ and $N_{\phi}$ denote the number of grids in altitude, latitude and longitude, respectively. While each voxel has a different volume, they have equal dimensions in global coordinates. The electron density profile is typically less dense at higher altitudes as shown in Fig. 1. It is also known as spatially correlated. Hence, higher altitude voxels having similar spatial distributions can be combined for more reliable reconstructions. 


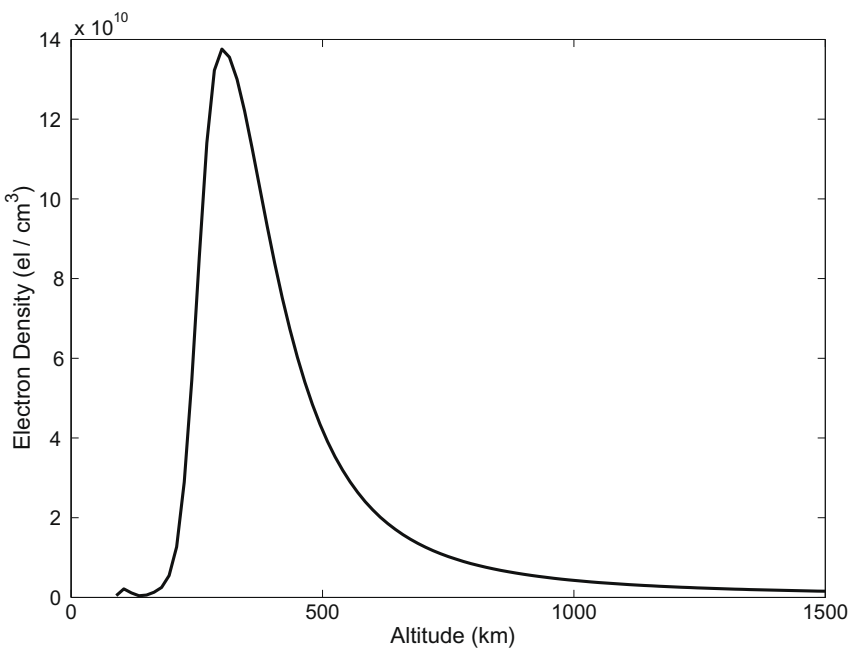

Fig. 1. The electron density profile as a function of altitude at $\theta=0^{\circ}$ and $\phi=0^{\circ}$ for July 15,2004 at 0200 UT.

An application of nonuniform voxelization in radial dimensions as a function of altitude is provided in Fig. 2. In order to find reconstruction of a day in a month, we need to provide a basis for that time of the day and day of the year. In this way, we will be able to adapt the basis to the conditions of the ionosphere at the time of interest. We form this basis by using SVD of a matrix whose columns are formed by the IRI-2007 profiles of $N_{d}$ days with similar sunspot numbers. Before the SVD computation, we vectorize the $3-\mathrm{D}$ voxels using $N_{r}, N_{\theta}$ and $N_{\phi}$. Spherical indices can be calculated as

$n_{r}=\left(r_{i}-90\right) / \Delta_{r}+1, \quad 1 \leqslant n_{r} \leqslant N_{r}$,

$n_{\phi}=\phi_{i} / \Delta_{\phi}+1, \quad 1 \leqslant n_{\phi} \leqslant N_{\phi}$,

$n_{\theta}=\theta_{i} / \Delta_{\theta}+1, \quad 1 \leqslant n_{\theta} \leqslant N_{\theta}$,

where $r_{i}, \phi_{i}$ and $\theta_{i}$ are the lower bounds of voxel $i$ in altitude, longitude and latitude, respectively. $\Delta_{\theta}, \Delta_{\phi}$ and $\Delta_{\theta}$ are the widths of voxels in altitude longitude and latitude,

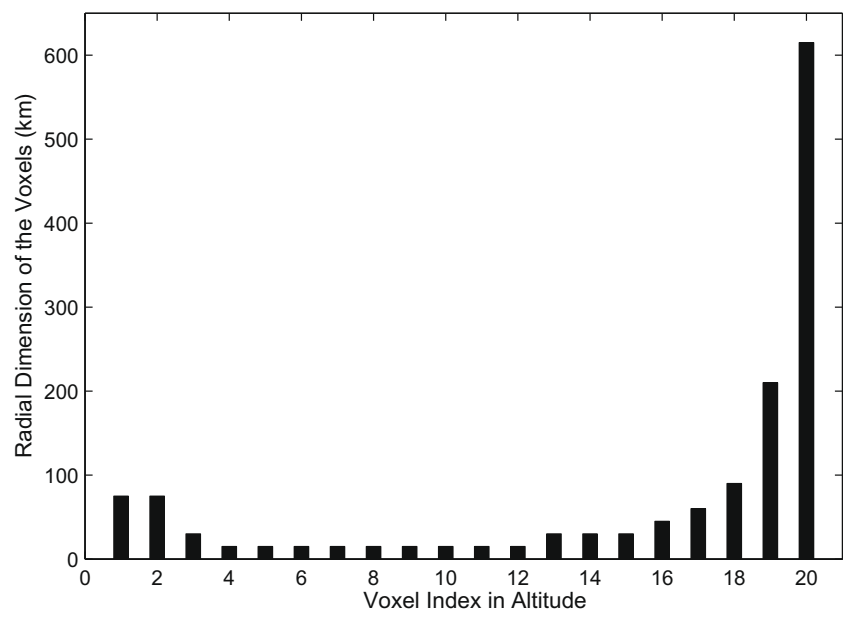

Fig. 2. Nonuniform voxelization of radial dimension as a function of altitude. respectively. In Eq. 3, $90 \mathrm{~km}$ represent the lower bound of the ionosphere. Then, electron density matrix in 3-D Space can be vectorized by

$\underline{e}_{d a y}(l)=e\left(n_{r}, n_{\phi}, n_{\theta}\right)$,

where index $l$ is related to $n_{r}, n_{\theta}$ and $n_{\phi}$ indices

$l=n_{r}+\left(n_{\phi}-1\right) N_{r}+\left(n_{\theta}-1\right) N_{r} N_{\phi}$.

Note that in Eq. 6, we suppressed the time dependence of the electron density distribution. Here we will first focus on reconstruction of the density distribution at a given time. Later in this section, we will incorporate the time variation to the reconstruction. Then, by obtaining electron density distribution models of IRI-2007 from different days with similar conditions, we form the following matrix $\mathbf{G}$, whose columns corresponds to data from individual days:

$\mathbf{G}=\left[\underline{e}_{d a y_{1}} \underline{e}_{d a y_{2}} \ldots \underline{e}_{d a y_{N_{d}}}\right]$.

We want to obtain a basis for the column spaces of this matrix. For this purpose Singular Value Decomposition (SVD) of $\mathbf{G}$ can be used:

$\mathbf{G}=\mathbf{U}_{N_{d}} \boldsymbol{\Sigma}_{N_{d}} \mathbf{V}_{N_{d}}^{H}$,

where columns of $\mathbf{U}_{N_{d}}$ form an orthogonal basis for the column space of $\mathbf{G}$, and $\boldsymbol{\Sigma}$ is a diagonal matrix:

$\boldsymbol{\Sigma}=\operatorname{diag}\left(\sigma_{1}, \sigma_{2}, \ldots, \sigma_{N_{d}}\right)$,

where the singular values are ordered in a decreasing order:

$\sigma_{1} \geqslant \sigma_{2} \geqslant \ldots \geqslant \sigma_{N_{d}} \geqslant 0$.

Although, columns of $\mathbf{U}_{N_{d}}$ form a basis, in practice, a subset that corresponds to the significant singular $\sigma$ values is sufficient. This is not only for reduction in the computational load, but also to introduce the required regularization for the reconstruction. In order to decide how many columns of $\mathbf{U}_{N_{d}}$ should be kept in the inversion process, we investigate the cumulative energy sequence defined below:

$E_{j}=\sum_{n=1}^{j} \sigma_{n}^{2}, \quad 1 \leqslant j \leqslant N_{d}$.

Out of $N_{d}$ basis, only $N_{s}$ significant basis are selected. The selected basis set contains more than $99 \%$ of the energy spectrum. An example is provided in Fig. 3 for July 2004 at 0200 UT where the first four basis components contains the $99.98 \%$ of all the energy. Then, in the reconstruction, we will model the electron density distribution as:

$\underline{e}=\mathbf{U}_{N_{s}} \underline{\alpha}$,

where $\alpha[i], 1 \leqslant i \leqslant N_{s}$ are the basis coefficients that should be estimated in the reconstruction process.

\subsection{3-D electron density reconstruction based on SVD basis}

For GPS data, we utilize data from each satellite and receiver combination that sees each other in a conical range of $D_{r}$ degrees that provides a single line integral as 


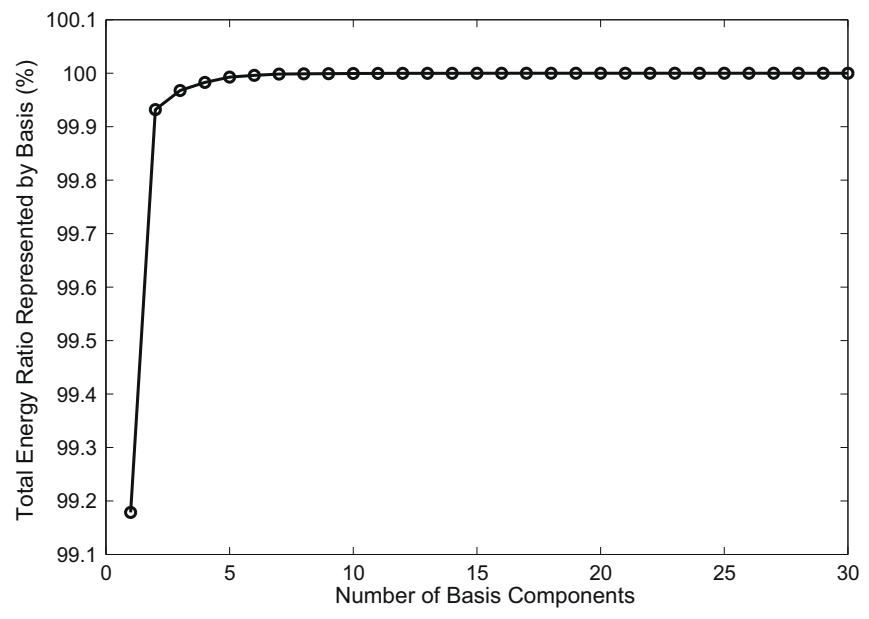

Fig. 3. The total energy captured by singular values as a function of their corresponding basis components for July 2004 at 0200 UT.

described in Eq. 1. For occultation data each LEO satellite pair that sees each other through ionosphere provides a line integral that can be used in the reconstruction. Assuming electron density profile remains about the same over a period of $15 \mathrm{~min}$, data in this window of $15 \mathrm{~min}$ can be combined to achieve 30 -fold increase in the available data for reconstruction. To investigate the accuracy of the inversion, in the reported results, we used IRI-2007 model result as the ground truth. Then, by using the actual satellite and receiver positions, we obtained synthetic STEC measurements, $y_{i}$, by computing the line integral given in Eq. 1 . In order to apply Eq. 1 on a voxelized ionosphere, we calculated the segments of the line that passes through each voxel. From the available measurements, a measurement vector $y$ is formed. In the proposed reconstruction technique, the measurement vector is related to the electron density in the 3-D voxelized structure as:

$\underline{y}_{N_{m}}=\mathbf{A}_{N_{m} \times N_{v}}{\underline{e_{N}}}_{N_{v}}$

where $N_{m}$ denotes the number of available measurements and $N_{v}$ denotes the number of voxels; $i$ th row of matrix A represents the length of line segments in each voxel that lies on the line connecting the receiver and the satellite corresponding to measurement $i$. Since electron density distribution can be expressed by basis components,

$\underline{e}_{N_{v}}=\mathbf{U}_{N_{v} \times N_{s}} \underline{\alpha}_{N_{s}}$,

Thus, the measurement model simplifies to the following form in terms of the unknown basis coefficients $\underline{\alpha}_{N_{s}}$

$\underline{y}_{N_{m}}=\mathbf{B}_{N_{m} \times N_{s}} \underline{\alpha}_{N_{s}}$

where the measurement matrix $\mathbf{B}=\mathbf{A U}_{N_{s}}$. This way, we can ease the computational load by decreasing the size of matrices drastically from $N_{m} \times N_{v}$ to $N_{m} \times N_{s}$. The unknown basis coefficients $\underline{\alpha}$ can be estimated as

$\hat{\alpha}=\arg \min _{\underline{\alpha}}\left((\underline{y}-\mathbf{B} \underline{\alpha})^{T} \mathbf{W}(\underline{y}-\mathbf{B} \underline{\alpha})\right)$, where $\mathbf{W}$ is a weight matrix of the measurements added within a time interval around the exact reconstruction time that puts more importance on the close-time and small angle measurements. The solution to the weighted least squares optimization problem in terms of the basis coefficients can be found as

$\underline{\hat{\alpha}}=\left(\mathbf{B}^{T} \mathbf{W B}\right)^{-1} \mathbf{B}^{T} \mathbf{W} \underline{y}$.

Hence 3-D electron density can be estimated by $\underline{\hat{e}}=\mathbf{U} \underline{\hat{\alpha}}$.

This reconstruction process is made for one time interval. We can compute the SVD basis components for each time slot, and provide reconstructions in a sliding window of time. This way we can obtain reconstruction of the electron density as a function of both space and time.

\section{Results}

The CIT technique discussed in this paper is applied for global ionospheric reconstruction. The focal point of this new CIT method discussed in the previous section relies on the fact that it uses SVD basis. In order to make a reconstruction based on this new method, we have to generate SVD basis offline. It is necessary to have a priori electron density profile for the construction of SVD basis. IRI2007 is a strong alternative in presenting a global electron density profile (International Reference Ionosphere, 2007) and it is used for generating SVD basis in this study. Electron density profiles with high space and time resolutions provide better accuracy in the reconstruction. In both simulations and reconstructions from GPS data, first the ionosphere have to be represented in terms of voxels as discussed in Section 2.2. The resolution sizes of the voxels are chosen according to the electron density variations in height, latitude and longitude in IRI-2007. In choosing the $\Delta_{r}$, we compared the magnitude of the electron density distribution in IRI-2007 for 5, 10, 15 and $45 \mathrm{~km}$. It is observed that $15 \mathrm{~km}$ is a reasonable distance in height since the difference in the magnitude of the electron densities between 5,10 and $15 \mathrm{~km}$ vertical resolutions are very small compared to the difference between 15 and $45 \mathrm{~km}$. Fifteen kilometers represents the significant variation in electron density profile of IRI-2007. Another comparison is made for $\Delta_{\phi}$ between $1^{\circ}, 2^{\circ}$ and $4^{\circ}$. The result is that an electron density profile with $\Delta_{\phi}=2^{\circ}$ is the best representation of the variations in IRI-2007. A similar investigation is also carried out for $\Delta_{\theta}$ where $2^{\circ}$ is selected as a result. In summary, for global ionospheric tomography, the voxel sizes are chosen as $\Delta_{r}=15 \mathrm{~km}, \Delta_{\phi}=2^{\circ}, \Delta_{\theta}=2^{\circ}$ where $90 \mathrm{~km} \leqslant$ $r \leqslant 1500 \mathrm{~km}, 0^{\circ} \leqslant \phi \leqslant 359^{\circ}$ and $-90^{\circ} \leqslant \theta \leqslant 90^{\circ}$ for altitude, longitude and latitude coordinates, respectively. This method is independent of time resolution so reconstructions can be made for any time resolution period. In order to generate the SVD basis, the electron density profiles $\underline{e}_{d a y}$ are obtained from IRI-2007 and $\mathbf{G}$ in Eq. 8 is formed. SVD is applied to $\mathbf{G}$ as discussed in Eq. 9 and the significant 
basis set $\mathbf{U}_{N_{s}}$ is chosen. An example set of $\mathbf{U}_{N_{s}}$ for the first four basis components are provided in Figs. 4 and 5 for the whole globe obtained for January and July 2004 at 0200, respectively. The figures show a cross-section of the basis at $390 \mathrm{~km}$ altitude which is the maximum ionization altitude of the ionosphere at $0^{\circ}$ latitude and $0^{\circ}$ longitude. As it can be observed from Fig. 4a and 5a, the highest energy of the basis is collected in the first basis component. Other basis components have significantly lower energy than the first basis component and the energy collected in the basis drops going from the first to the last basis. As it can be observed, with only four basis the globe can be represented.

While SVD basis clearly decreases the computational complexity of the reconstruction by allowing a global CIT with only four basis, it has also an important physical meaning. SVD basis change with respect to the hour of the day and day of the year. Figs. 4a and 5a show the change of the basis with respect to seasons. While the peak point of the July basis shown in Fig. 5a is at the northern hemisphere near the tropic of cancer, the peak point of the January basis shown in Fig. 4a is at the southern hemisphere near the tropic of capricorn. In our simulations, it has also been seen that peak point of the basis rotates with respect to time following the Sun.

The CIT reconstruction technique discussed in this study is first applied to simulations based on IRI-2007 model. For these simulations, synthetic STEC measurements are calculated from IRI-2007 electron density profile. These synthetic measurements are calculated between 56 receivers and 29 satellites obtained from International GPS Service (2007). The receivers used in the simulations are shown in Fig. 6. A synthetic STEC measurement, $y_{r_{n}, s_{m}}\left(t_{0}\right)$ is obtained by line integral of the electron density values along the line that join the GPS station and satellite at that given time using Eq. 1. The collection of STEC mea- surements form $y$ in Eq. 18. Using synthetic TEC measurements provide ${ }^{-}$certain advantages. There are no measurement errors and they are calculated directly from IRI-2007 which provides a reconstruction refined from other error sources except the method itself. Since synthetic TEC measurements fit the SVD basis best, it forms a minimum error bound for the method proposed. Synthetic measurements also enables measurements to be obtained for regions where real data cannot be collected or very sparse like oceans and poles.

For each simulation, the reconstruction and the IRI2007 model are compared and a normalized reconstruction error is found using the equation:

$R_{e}=\|\underline{e}-\underline{\hat{e}}\| /\|\underline{e}\|$,

where $\underline{e}$ is defined in Eq. 13 and $\underline{\hat{e}}$ is given in Eq. 19. By using synthetic TEC measurements, we reconstructed ionosphere for January 15 and July 15, 2004 at 0200 UT. Reconstructed ionospheres in Fig. 7a (January) and 7c (July) are very close estimates to the IRI-2007 models in Fig. $7 \mathrm{~b}$ (January) and $7 \mathrm{~d}$ (July) where the respective normalized reconstruction errors are 0.0586 and 0.0663 , respectively. Another comparison of reconstructed ionosphere with IRI-2007 model is made for July 15, 2004 at 0000 UT. The normalized error of reconstruction for this case is 0.0822 . To investigate the performance of the reconstruction on noisy STEC measurements, we added independent identically distributed random noise with zero mean and a standard deviation equal to the $25 \%$ of the mean of STEC values on the synthesized STEC measurements. Then, we performed reconstruction on the noisy STEC measurements. Fig. 8a shows the reconstruction from noisy STEC measurements. The calculated normalized error is 0.0712 which is very slightly higher than the noise-free reconstruction error which is 0.0586 . This shows that SVD based technique is very robust to noise. Using the

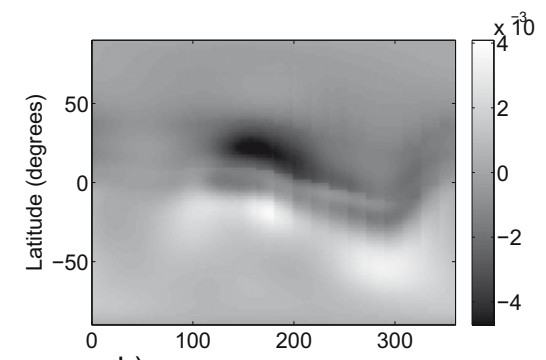

b) Latitude (degrees)

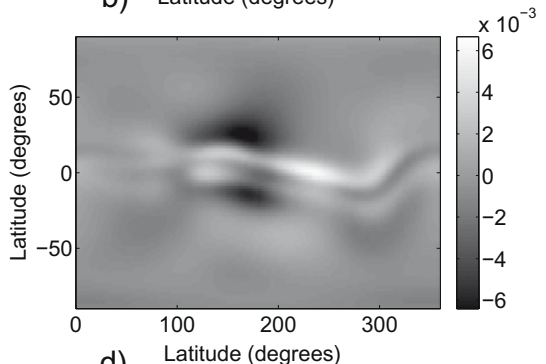

d)

Fig. 4. The first four basis components for January 15, 2004 at 0200 UT at $390 \mathrm{~km}$ altitude. (a) First basis, (b) second basis, (c) third basis, (d) fourth basis. 

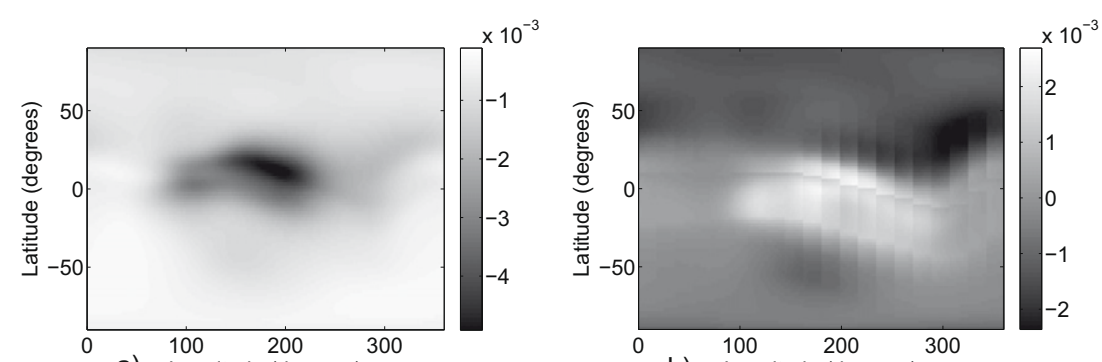

a) Longitude (degrees)
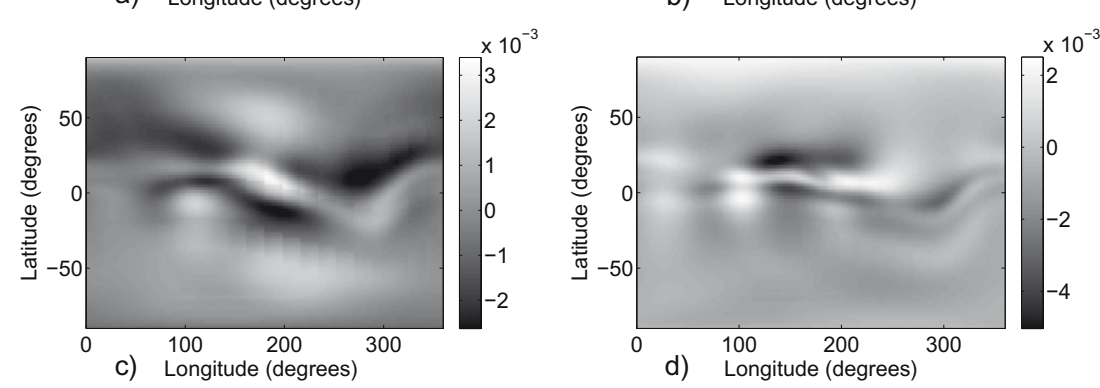

Fig. 5. The first four basis components for July 15, 2004 at 0200 UT at $390 \mathrm{~km}$ altitude. (a) First basis, (b) second basis, (c) third basis, (d) fourth basis.

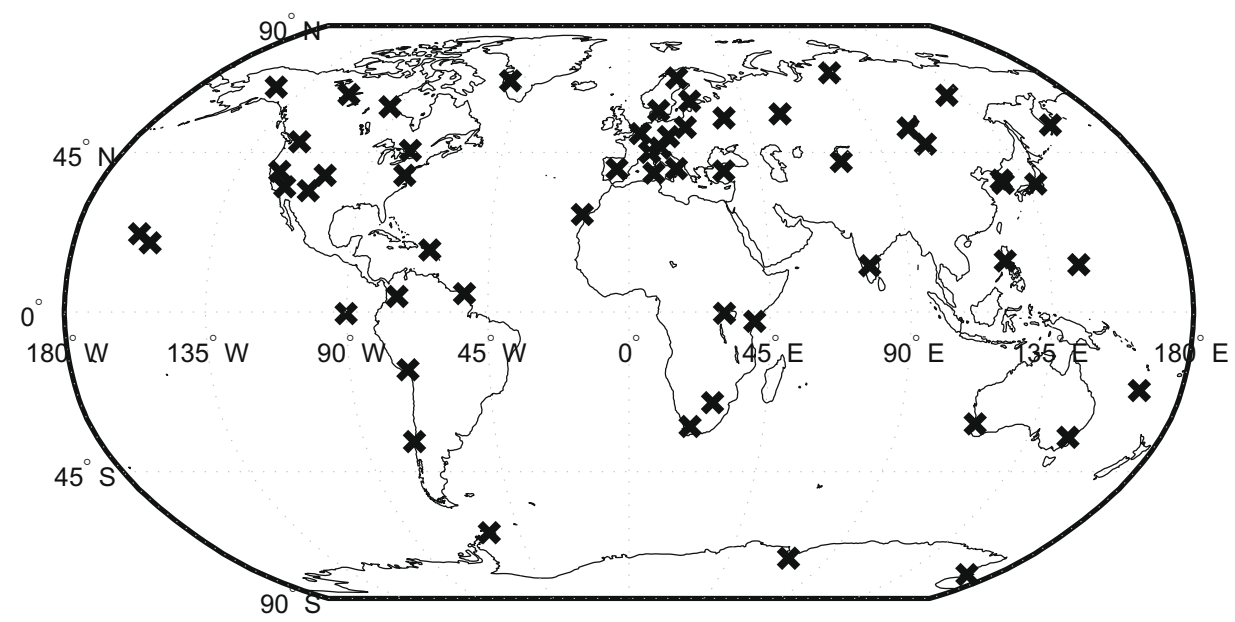

a)

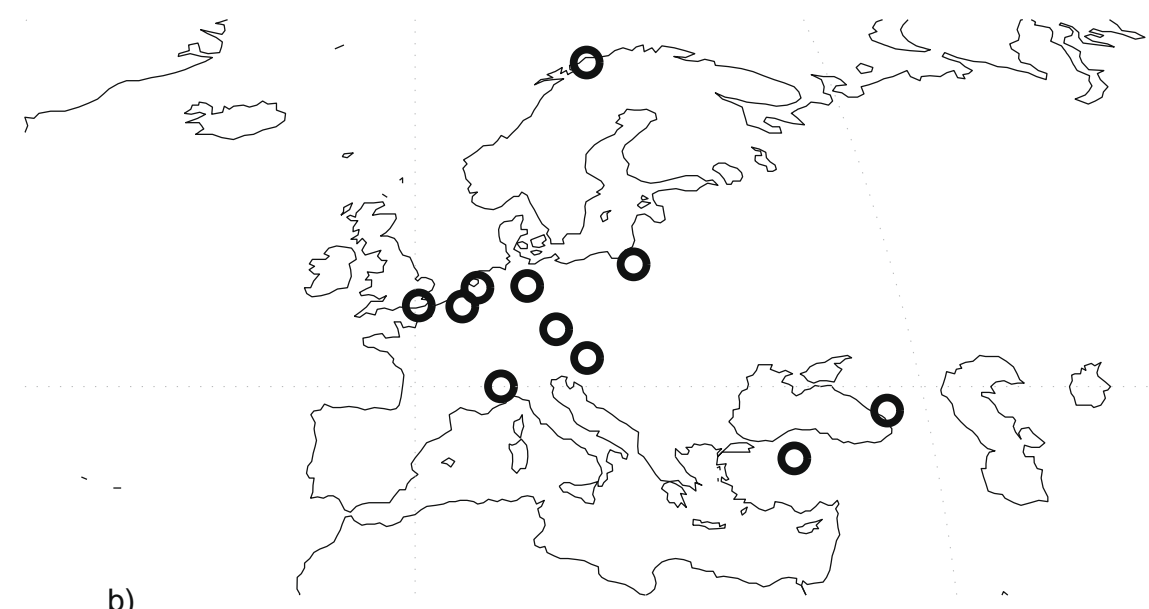

Fig. 6. Locations of the GPS receivers used in the reconstructions. (a) Receivers used for deriving synthetic measurements, (b) 11 receivers that are used in GPS-TEC measurements. 


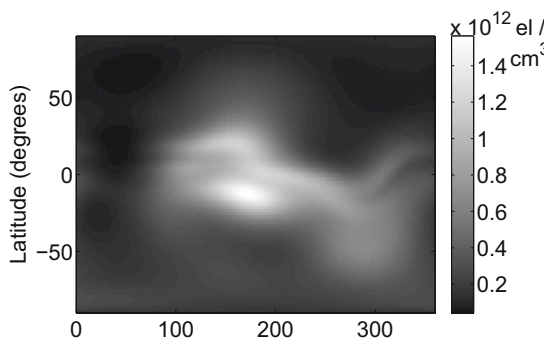

a) Longitude (degrees)

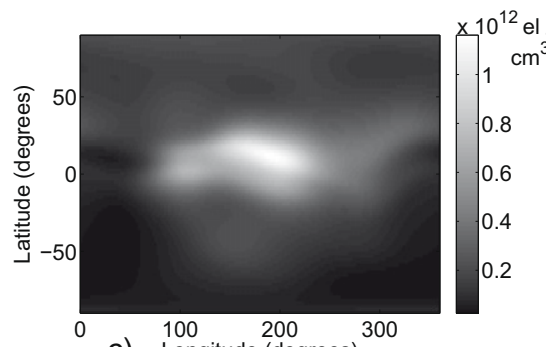

c) Longitude (degrees)

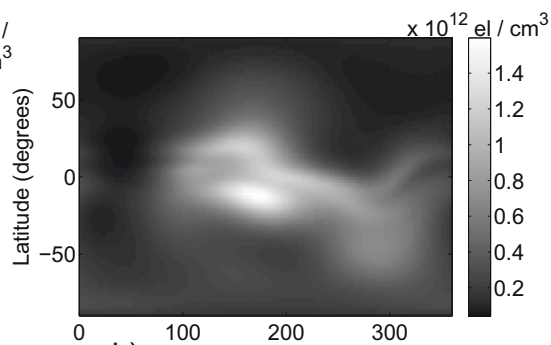

b) Longitude (degrees)

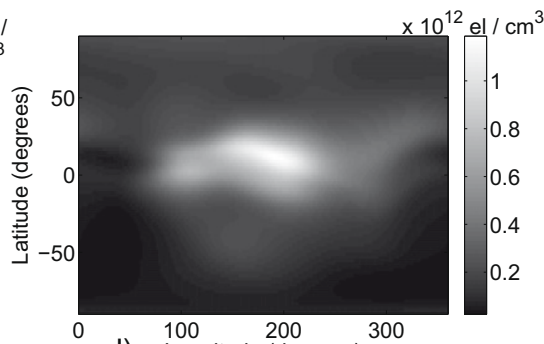

d) Longitude (degrees)

Fig. 7. Comparison of the IRI-2007 model with SVD-based CIT from synthetic data at 0200 UT at $390 \mathrm{~km}$ altitude. (a) Reconstruction for January 15 , 2004, (b) IRI-2007 Model for January 15, 2004, (c) Reconstruction for July 15, 2004, (d) IRI-2007 Model for July 15, 2004.
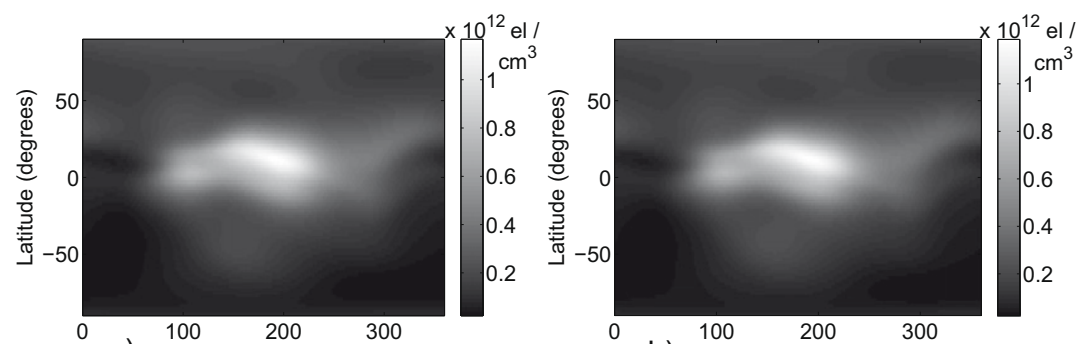

a) Longitude (degrees)

b) Longitude (degrees)
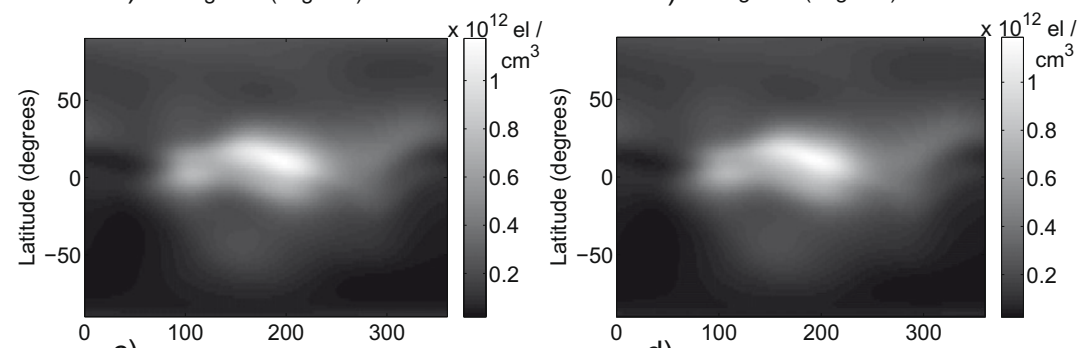

C) Longitude (degrees)

d) Longitude (degrees)

Fig. 8. Comparison of the IRI-2007 model with SVD-based CIT from noisy synthetic data and perturbed ionosphere for July 15 , 2004 at 0200 UT at $390 \mathrm{~km}$ altitude. (a) Reconstruction from noisy synthetic data, (b) IRI-2007 Model, (c) reconstruction from perturbed ionosphere (d) IRI-2007 Model.

same technique we used to obtain synthetic STEC measurements from IRI-2007 model, a second set of STEC measurements between ankr receiver and available satellites are derived from the reconstructed ionosphere. The normalized error between initial noise-free STEC measurements and second set of STEC measurements obtained from reconstruction is 0.0077 . We can consider the noisy STEC measurements we produced before as a third set of measurements. A fourth set of STEC measurements can be derived from the ionosphere reconstructed from noisy STEC measurements. The normalized error between noisy STEC measurements and the fourth set of STEC measurements is only 0.1348 . This shows that the reconstruction method is very robust to noise.
In order to observe the robustness of the new SVDbased CIT technique on the possible perturbation of the IRI-2007 model, the original model is modified to include variations. For this purpose, the 3-D electron distributions are again obtained from the IRI model. Then, the model $\underline{e}$ is multiplied by a perturbation distribution $\underline{\gamma}$ and a new distribution $\underline{e_{p}}$ is obtained as:

$\underline{e_{p}}(r, \phi, \theta)=\underline{e}(r, \phi, \theta) \cdot \underline{\gamma}(r, \phi, \theta)$,

where $\underline{\gamma}(r, \phi, \theta)$ is a perturbation array. The entries in $\underline{\gamma}$ are chosen as samples of a realization of a stationary 3 -D random field with the following parameters: $E\{\gamma(r$, $\phi, \theta)\}=1, \operatorname{Var}(\underline{\gamma}(r, \phi, \theta))=E\left\{(\underline{\gamma}(r, \phi, \theta)-1)^{2}\right\}=0.16$, 
Table 1

GPS Receivers and Coordinates.

\begin{tabular}{lllcl}
\hline City & Country & Station ID & Longitude & Latitude \\
\hline Brussels & Belgium & brus & 4.35 & 50.79 \\
Graz & Austria & graz & 15.49 & 47.06 \\
Hailsham & England & hers & 0.33 & 50.86 \\
Torino & Italy & ieng & 7.64 & 45.01 \\
Kootwijk & Netherlands & kosg & 5.81 & 52.18 \\
Olsatyn & Poland & lama & 20.67 & 53.89 \\
Braunschweig & Germany & ptbb & 10.46 & 52.30 \\
Tromsoe & Norway & tro1 & 18.94 & 69.66 \\
Bad Koetzting & Germany & wtzr & 12.88 & 49.14 \\
Zelenchukskaya & Russia & zeck & 41.56 & 43.29 \\
Ankara & Turkey & ankr & 32.76 & 39.89 \\
\hline
\end{tabular}

$E\left\{(\gamma(r, \phi, \theta)-1)\left(\gamma\left(r+\Delta_{r}, \phi+\Delta_{\phi}, \theta+\Delta_{\theta}\right)-1\right)\right\}=$ $0.1 \bar{\sigma}_{r}\left(\Delta_{r}\right) f_{\phi}\left(\Delta_{\phi}\right) f_{\theta}\left(\bar{\Delta}_{\theta}\right)$, where $E$ is the expectation operator, $V a r$ is the variance and $f_{r}\left(\Delta_{r}\right)=1-\Delta_{r} / 1410 \mathrm{~km}$, $f_{\phi}\left(\Delta_{\phi}\right)=1-\Delta_{\phi} / 360^{\circ}$ and $f_{\theta}\left(\Delta_{\theta}\right)=1-\Delta_{\theta} / 180^{\circ}$. By choosing the random field as a spatially correlated one, we can perturb the IRI-2007 model in a spatially correlated way. By using the perturbed model, we achieved a reconstruction with error 0.0730 which also proves that SVDbased reconstruction can be used where the actual ionosphere deviates from the IRI-2007 model. Fig. 8c and 8d show the reconstruction from the perturbed ionosphere at July 15, 2004 at $0200 \mathrm{UT}$ at $390 \mathrm{~km}$ altitude and the IRI2007 model.

The new SVD-based CIT technique is also tried with experimental data obtained from GPS-TEC. From the 56 receiver locations given in Fig. 6a, only 11 of these are used for the reconstruction. These 11 receivers are indicated by circles on Fig. 6b. The list of these receivers and their coordinates are also provided in Table 1. The STEC from the GPS receivers are obtained using Reg-Est and IonolabTEC as discussed in detail in Nayir et al. (2007) and $y$ in Eq. 18 is formed. The SVD basis obtained as describe in
Table 2

$M_{e}$, for cross-validation for the reconstructions using GPS-TEC.

\begin{tabular}{ll}
\hline Station ID - Date - Hour (UT) & $M_{e}$ \\
\hline brus, July 15,2004 at 0200 & 1.3819 \\
brus, July 15,2004 at 0000 & 1.2159 \\
graz, January 15,2004 at 0200 & 1.5117 \\
graz, July 15, 2004 at 0200 & 1.8763 \\
graz, July15, 2004 at 0000 & 0.8742 \\
ptbb, January 15,2004 at 0200 & 1.5505 \\
ptbb, July 15, 2004 at 0200 & 1.7141 \\
ptbb, July15, 2004 at 0000 & 1.7316 \\
\hline
\end{tabular}

Eq. (9) and (13) from IRI-2007 model is used in reconstructions and $\underline{\hat{e}}$ in Eqs. (18) and (19) is formed. In Fig. 9a and $9 \mathrm{c}$, examples for reconstruction are provided for January 15, 2004 and July 15, 2004, respectively, at 0200 UT at $390 \mathrm{~km}$ altitude. As it can be observed from Fig. 9, the reconstruction for the whole globe in latitude, longitude and altitude with only 11 GPS receivers is very successful compared to IRI-2007 model.

In order to cross-validate our results, STEC measurements from one of the receivers is left out as control data and the reconstruction is based on the remaining 10 receivers. For this purpose, STEC measurements are derived from the reconstructions obtained using 10 receivers and compared with the original STEC measurements for the control receiver. The cross-validation is checked for three different receivers, namely, graz, ptbb and brus for different dates and times. Table 2 shows mean square error

$M_{e}\left(u, t_{0}\right)=\sqrt{\frac{1}{M} \sum_{m=1}^{M}\left(\hat{y}_{u, m}\left(t_{0}\right)-y_{u, m}\left(t_{0}\right)\right)^{2}}$,

where $\hat{y}_{u, m}\left(t_{0}\right)$ denotes the STEC obtained from reconstructed ionosphere and $y_{u, m}\left(t_{0}\right)$ denotes the STEC from GPS measurements for receiver $u$ and satellite $m$ at time

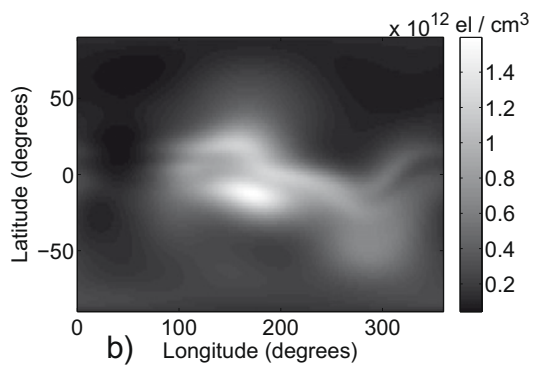

a) Longitude (degrees)
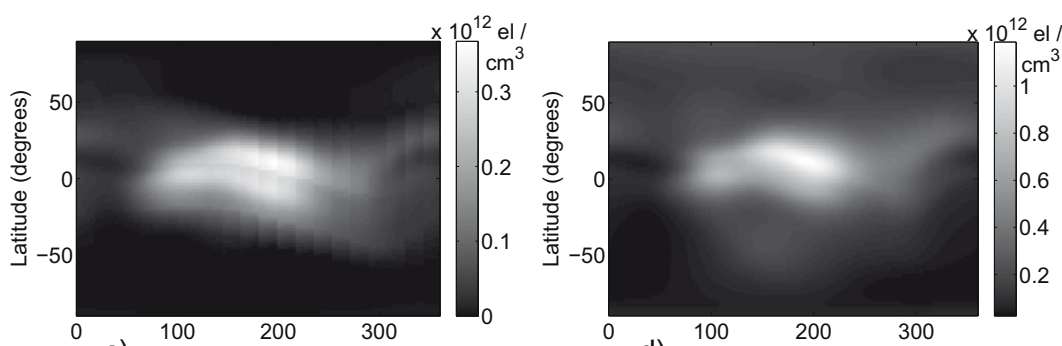

d) $\quad 100 \stackrel{200}{20} \stackrel{300}{ }$

Fig. 9. Comparison of the IRI-2007 model with SVD-based CIT from GPS-TEC measurements at 0200 UT at $390 \mathrm{~km}$ altitude. (a) Reconstruction for January 15, 2004, (b) IRI-2007 Model for January 15, 2004, (c) reconstruction for July 15, 2004, (d) IRI-2007 Model for July 15 , 2004. 
instant $t_{0} . M$ is the total number of satellites within the view of receiver $u$ at time $t_{0}$. The mean square error values provided in Table 2 indicate the high performance of the new CIT technique. For all receivers and date, $M_{e}$ is less than 2 TECU.

The results presented in this section denotes the success of the SVD-based CIT reconstruction technique both over simulated model data and experimental GPS-TEC data. The SVD basis not only represents the underlying ionosphere very well but also reduces the computational complexity significantly.

\section{Conclusions}

In the reconstruction, the 3-D ionospheric electron density distribution is modeled as a linear combination of few appropriately chosen basis components. To obtaine the most representetive basis components, IRI-2007 results obtained with the similar conditions, e.g., time of the day and solar activity, are analyzed by using Singular Value Decomposition (SVD). It is observed that the first 4 or 5 SVD basis components dominates the rest significantly. Thus, in the resonstruction, the ionospheric electron density distribution is assumed to be in the span of these few basis components, resulting in a very efficient and robust density distribution estimates. We provided reconstruction results based on both synthetic and real GPS based TEC measurement data. For the validation of the reconstructions on the real data, cross-validation approach indicated that the reconstruction error is about $1.50 \mathrm{TECU}$. The reconstruction technique can also utilize occultation measurements. It is expected that the accuracy of the reconstructions will improve further when the GPS based TEC measurements and occultation data are used together. Our current research is focused on this investigation. We will report results if this highly promising investigation is near future.

\section{Acknowledgement}

This work is supported by TÜBITAK EEEAG Grant $105 \mathrm{E} 171$.

\section{References}

Arikan, F., Erol, C.B., Arikan, O. Regularized estimation of vertical total electron content from global positioning system data. J. Geophys. Res. 108 (A12), 1469-1480, 2003.

Arikan, F., Erol, C.B., Arikan, O. Regularized estimation of VTEC from GPS data for a desired time period. Radio Sci. 39 (6), RS6012, 2004.

Arikan, F., Arikan, O., Erol, C.B. Regularized estimation of TEC from GPS data for certain midlatitude stations and comparison with the IRI model. Adv. Space Res. 39, 867-874, 2007.

Arikan, O., Arikan, F., Erol, C.B. 3-D Ionospheric Tomography with Random Field Priors Mathematical Methods in Engineering, in: Tas, K., Tenreiro Machado, J.A., Baleanu, D. (Eds.). Springer, Netherlands, pp. 334-335, 2007a.

Arikan, O., Arikan, F., Erol, C.B. Computerized ionospheric tomography with the IRI model. Adv. Space Res. 39, 859-866, $2007 \mathrm{~b}$.

Austen, J.R., Franke, S.J., Liu, C.H. Ionospheric image using computerized tomography. Radio Sci. 23, 299-307, 1988.

Garcia Fernandez, M., Hernandez-Pajares, M., Juan, M., Sanz, J. Improvement of ionospheric electron density estimation with GPSMET occultations using Abel inversion and VTEC information. $\mathbf{J}$. Geophys. Res. 108, 1338, $2003 \mathrm{a}$.

Garcia Fernandez, M., Hernandez-Pajares, M., Juan, M., Sanz, J., Orus, R., Coisson, P., Nava, B., Radicella, S.M. Combining ionosonde with ground GPS Sata for electron density estimation. J. Atmos. Sol. Terr. Phys. 65, 683-691, 2003b.

Hajj, G.A., Romans, L.J. Ionospheric electron density profiles obtained with GPS results from the GPS/MET experiment. Radio Sci. 33, 175190, 1998.

Hansen, A.J., Walker, T., Enge, P. Ionospheric correction using tomography. Proc. Inst. Nav. GPS 97, 249-257, 1997.

International GPS Service, Available from: http://igs.ens.ign.fr.

International Reference Ionosphere, Available from: <http:// iri.gsfc.nasa.gov>.

IONOLAB, Available from: $<$ http://www.ionolab.org/>.

Jakowski, N., Sardon, E., Engler, E., Jungstand, A., Klahn, D. Relationships between GPS-signal propagation errors and EISCAT observations. Ann. Geophysicae 14, 1429-1436, 1996.

Komjathy, A. Global Ionospheric Total Electron Content Mapping Using the Global Positioning System, Ph.D. Thesis, Dept. of Geodesy and Geomatics Engineering Technical Report No. 188, Univ. of New Brunswick, Fredericton, New Brunswick, Canada, 1997.

Liao, X., Carrier phase based ionosphere recovery over a regional area GPS network, M.Sc. Thesis, Univ. of Calgary, Canada, 2000.

Nayir, H., Arikan, F., Arikan, O., Erol, C.B. Journal of Geophysical Research 112, A11313, doi:10.1029/2007JA012459, 2007.

Pryse, S.E., Kersley, L. A preliminary experimental test of ionospheric tomography. J. Atmos. Terr. Phys. 54, 1007-1012, 1992.

Tsai, L.C., Tsai, W.H., Schreiner, W.S., Berkey, F.T., Liu, J.Y. Comparisons of GPS/MET retrieved ionospheric electron density and ground based ionosonde data. Earth Planets Space 53, 193-205, 2001. 